\title{
Detecção da Doença de Alzheimer em Diferentes Estágios através do Espectro de Potências da Wavelet Packet de Sinais EEG
}

\author{
Rui M. da Cunha, Bruno C. Bispo e Pedro M. Rodrigues
}

\begin{abstract}
Resumo-Este trabalho tem como objetivo detectar a doença de Alzheimer em diferentes estágios através de sinais EEG. Potências relativas às frequências convencionais são obtidas dos valores máximo, mínimo e médio de estimativas do espectro de potências da Wavelet Packet. Para cada par de grupos de estudo e eletrodo, uma seleção dos parâmetros é realizada. Os parâmetros selecionados são utilizados como entrada para classificadores com validação cruzada leave-one-out. Acurácias de classificação de $100 \%$ são obtidas, em pelo menos 1 eletrodo, para os 6 pares de grupos analisados, indicando as regiões no escalpe com maiores diferenças à medida que a doença progride. $E$ acurácias médias, incluindo todos os eletrodos, entre 81,3 e $91,4 \%$ são alcançadas.
\end{abstract}

Palavras-Chave-Doença de Alzheimer, EEG, espectro de potência, sub-banda, wavelet packet.

Abstract-This work aims to detect the Alzheimer's disease at different stages using EEG signals. Powers related to conventional frequencies are obtained from the maximum, minimum and average values of Wavelet Packet power spectrum estimates. For each pair of study groups and electrodes, a parameter selection is performed. The selected parameters are used as input for classifiers with leave-one-out cross-validation. Classification accuracies of $100 \%$ are obtained, in at least 1 electrode, for the 6 pairs of groups analyzed, indicating the regions in the scalp with the greatest differences as the disease progresses. Average classification accuracies, including all electrodes, between 81.3 and $91.4 \%$ are achieved.

Keywords-Alzheimer disease, EEG, power spectrum, subband, wavelet packet.

\section{INTRODUÇÃo}

A doença de Alzheimer (DA) é um distúrbio crônico e neurodegenerativo que progressivamente atinge todas as funções cerebrais [1]. É a doença neurodegenerativa com maior incidência mundial, afetando cerca de 46 milhões de pessoas [2]. O risco de desenvolver DA é de $1 \%$ em pessoas com idade entre 60 e 65 anos e cerca de $40 \%$ acima dos anos 85 [3].

Até o presente momento, DA não tem cura [4]. Existem soluções que apenas podem atrasar a sua progressão. Portanto, a pesquisa por métodos para detectar DA em estágios iniciais é de extrema importância para retardar a progressão e os sintomas da mesma, contribuindo assim para uma melhor qualidade e maior expectativa de vida dos pacientes.

A evolução da DA é dividida em quatro estágios dependentes dos sintomas apresentados pelo paciente: défice

Rui M. da Cunha e Pedro M. Rodrigues, Universidade Católica Portuguesa, CBQF - Centro de Biotecnologia e Química Fina - Laboratório Associado, Escola Superior de Biotecnologia, Porto, Portugal, e-mail: prodrigues@porto.ucp.pt; Bruno C. Bispo, Departamento de Engenharia Elétrica e Eletrônica, Universidade Federal de Santa Catarina, Florianópolis-SC, Brasil, e-mail: bruno.bispo@ufsc.br. cognitivo ligeiro (MCI, do inglês Mild Cognitive Impariment); leve; moderado; avançado. O MCI é o estágio pré-demência onde os sintomas são pouco evidentes, normalmente lapsos esporádicos de memória que são entendidos pelo paciente como uma consequência normal da idade ou atribuídos ao cansaço, e o paciente consegue viver de forma independente [5], [6]. É a fase de transição entre o envelhecimento normal e a patologia. Pacientes nesse estágio podem ou não desenvolver DA, ocorrendo de 10 a $15 \%$ dos casos [7].

No estágio leve, as memórias episódica e semântica começam a se deteriorar e o prejuízo se torna perceptível [8]. O estágio moderado é onde o comprometimento cognitivo se torna evidente. Julgamento, planejamento, organização e raciocínio lógico ficam prejudicados. As tarefas diárias se tornam uma luta, levando a perda da independência e necessidade de monitoramento [5], [8]. Questionamentos são frequentes e delírios podem ocorrer [8]. Neste trabalho, pacientes nos estágios leve e moderado são considerados um único grupo de estudo, denominado ADM, como normalmente ocorre na literatura. No estágio avançado (ADA), quase todas as funções cognitivas e motoras são afetadas e, consequentemente, o paciente precisa de cuidado em tempo integral [5], [6], [8].

O diagnóstico definitivo da DA somente pode ser realizado após a morte do paciente [9]. No entanto, algumas ferramentas são utilizadas para auxiliar o diagnóstico. Uma vez que as capacidades cognitivas vão se deteriorando à medida que a degeneração neural progride, exames que medem a capacidade física e cognitiva dos pacientes são comumente utilizados em ambientes clínicos para avaliar a evolução da DA. O mais comum é o mini exame do estado mental (MMSE, do inglês Mini Mental State Exam), que consiste de perguntas com o objetivo de avaliar a capacidade de orientação, atenção, memória e linguagem do paciente, com escala de 0 a 30 [10].

Além disso, diversos equipamentos médicos estão disponíveis para avaliar diferentes marcadores biológicos relacionados com DA, como ressonância magnética, tomografia por emissão de pósitrons, tomografia computadorizada por emissão de fóton único, magnetoencefalografia, análise de sangue e do fluído cerebrospinal, e a eletroencefalografia (EEG) [11]. Entre esses, a EEG destaca-se por ser amplamente utilizada em ambientes clínicos devido ao seu sistema de gravação ser barato, não-invasivo, móvel e rápido [12].

Alguns trabalhos sobre deteç̧ão de DA através de sinais EEG são encontrados na literatura. Esses trabalhos focam na classificação binária entre um ou mais pares de grupos, os quais incluem pacientes diagnosticados com DA em diferentes 
TABELA I

INFORMAÇÃO DA BASE DE DADOS.

\begin{tabular}{c|c|c|c}
\hline Grupo & No. de indivíduos & Idade média & MMSE médio \\
\hline \hline Controle (C) & 11 & 74 & 28,68 \\
MCI & 8 & 80 & 26,29 \\
ADM & 11 & 79 & 18,89 \\
ADA & 8 & 79 & 11,50 \\
\hline
\end{tabular}

estágios e indivíduos saudáveis (controle). Por motivos de organização do texto, as informações referentes ao estado-daarte, nomeadamente pares de grupos e acurácias de classificação, são mostrados na tabela presente no final do documento, juntamente com os resultados médios obtidos nesse trabalho.

Esse trabalho tem como objetivo detectar a doença de Alzheimer em diferentes estágios através de sinais EEG. Com intuito de explorar uma possível maior capacidade discriminante de bandas frequenciais, potências relativas às frequências convencionais são obtidas dos valores máximo, mínimo e médio de estimativas do espectro de potências da Wavelet Packet. O artigo está organizado da seguinte maneira: a Seção II descreve a base de EEG utilizada; a Seção III explica a decomposição multibanda realizada e as potências relativas utilizadas; a Seção IV apresenta e discute os resultados obtidos; e, por fim, a Seção V conclui o trabalho.

\section{BASE DE DADOS DE EEG}

A base de dados consiste de 38 exames de EEG gravados no Hospital São João, Porto, Portugal, projeto CES198-14, com a aprovação do comitê de ética local e do conselho de administração do hospital. Os sinais foram adquiridos a uma frequência de amostragem de $256 \mathrm{~Hz}$ através de 19 eletrodos colocados sobre o escalpe de acordo com o sistema 10-20, resultando em 19 sinais por exame (indivíduo). Os indivíduos encontravam-se num estado de relaxamento e com os olhos fechados. Entre os indivíduos, 11 são saudáveis (controle), 8 com MCI, 11 com ADM e 8 com ADA. O MMSE e a idade média dos grupos de estudo estão apresentados na Tabela I.

Todos os sinais foram filtrados usando um filtro passa-banda digital com frequências de corte de 1 e $40 \mathrm{~Hz}$, faixa frequencial convencional de EEG, a fim de remover artefatos e ruído elétrico. A amplitude de cada sinal foi normalizada segundo

$$
x(n)=\frac{x(n)}{\sum_{n=0}^{N-1} x^{2}(n)},
$$

onde $N$ representa o tamanho dos sinais, e a componente contínua foi removida. Por fim, cada sinal foi segmentado em 20 trechos não-sobrepostos com duração de $5 \mathrm{~s}$.

\section{ParÂmetros Espectrais Via WaVElet}

\section{A. Decomposição Multibanda com Wavelet Packet}

A transformada wavelet discreta (DWT, do inglês Discrete Wavelet Transform) de um sinal de tempo discreto e energia finita é a sua decomposição em bandas de oitavas, portanto não-uniformes, a partir de um par de filtros de meia-banda [13]. Após a decomposição do sinal pelo par de filtros, a banda passa-baixa é decomposta sucessivamente até o nível desejado.

A transformada wavelet packet discreta (DWPT) é uma extensão da DWT onde as bandas passa-baixa e passa-alta do sinal são sucessivamente e igualmente decompostas, resultando numa decomposição uniforme do sinal a partir do par de filtros meia-banda [13].

Os sinais resultantes dessa decomposição sucessiva com estrutura em árvore são definidos como

$$
W_{j, p}(n)= \begin{cases}\sqrt{2^{j}} \sum_{k=0}^{N-1} h_{0}(2 n-k) W_{(j-1),(p / 2)}(k), & \text { se } p \text { par } \\ \sqrt{2^{j}} \sum_{k=0}^{N-1} h_{1}(2 n-k) W_{(j-1),(p-1 / 2)}(k), & \text { se } p \text { ímpar }\end{cases}
$$

onde $j$ é o nível de decomposição, $N$ é o tamanho dos sinais, $p=0,1, \ldots, 2^{j}-1$ é a posição na árvore no nível $j, h_{0}(n)$ e $h_{1}(n)$ são os filtros passa-baixa e passa-alta de meia banda. Ressalta-se que $W_{0,0}(n)$ é o sinal original, o sinal de sub-banda $W_{j, p}(n)$ tem conteúdo frequencial limitado em $\left[p \pi / 2^{j},(p+1) \pi / 2^{j}\right]$, onde $\pi$ é a frequência angular normalizada, e $h_{0}(n)$ e $h_{1}(n)$ dependem da wavelet utilizada.

Neste trabalho, a DWPT foi aplicada até o décimo nível $(j=10)$ aos 20 segmentos dos 19 canais de cada indivíduo. Os sinais das sub-bandas $W_{10, p}(n)$ foram reamostrados para a frequência de amostragem original por meio de interpolação.

\section{B. Espectro de Potência}

O espectro da DWPT de $x(n)$ pode ser definido como [14]

$$
S(n, p)=\left|W_{10, p}(n)\right|^{2},
$$

onde $n=0,1, \ldots, N-1$ e $p=0,1, \ldots, 2^{10}-1$. Para $n$ fixo, $S(n, p)$ pode ser entendido como uma estimativa com 1024 pontos do espectro de potência do sinal no instante $n$. Por esse motivo, $S(n, p)$ também é chamado de escalograma em analogia ao espectrograma [15].

Com o intuito de caracterizar o comportamento dessas estimativas do espectro de potência, calculou-se os seus valores máximo, mínimo e médio ao longo do tempo, resultando em

$$
\begin{gathered}
P S_{\text {max }}(p)=\max _{n}\{S(n, p)\}, \\
P S_{\text {min }}(p)=\min _{n}\{S(n, p)\}
\end{gathered}
$$

e

$$
P S_{\text {med }}(p)=\frac{1}{N} \sum_{n=0}^{N-1} S(n, p) .
$$

E em seguida os valores são normalizados da seguinte forma

$$
P S_{i}(p)=\frac{P S_{i}(p)}{\sum_{p=0}^{1023} P S_{i}(p)},
$$

onde $i=\{\max , \min , \operatorname{med}\}$.

\section{Potências Relativas}

Os sinais de EEG são tipicamente divididos nas bandas frequenciais $\delta(1-4 \mathrm{~Hz}), \theta(4-8 \mathrm{~Hz}), \alpha(8-13 \mathrm{~Hz}), \beta(13-30 \mathrm{~Hz})$ e $\gamma(30-40 \mathrm{~Hz})$. E há indícios que a DA gera um deslocamento energético das altas para as baixas frequências [16].

Por isso, decidiu-se calcular a potência de $P S_{\max }(p)$, $P S_{\text {min }}(p)$ e $P S_{\text {med }}(p)$ relativas às sub-bandas convencionais 
de EEG. Isso é realizado ao somar os seus valores nos índices $p$ correspondentes a cada sub-banda, isto é,

$$
\begin{aligned}
R P_{\delta, i} & =\sum_{p=0}^{32} P S_{i}(p), \\
R P_{\theta, i} & =\sum_{p=32}^{64} P S_{i}(p), \\
R P_{\alpha, i} & =\sum_{p=64}^{104} P S_{i}(p), \\
R P_{\beta, i} & =\sum_{p=104}^{240} P S_{i}(p), \\
R P_{\gamma, i} & =\sum_{p=240}^{320} P S_{i}(p),
\end{aligned}
$$

onde $i=\{\max , \min , \operatorname{med}\}$.

\section{Metodologia e Resultados}

Para cada um dos 38 indivíduos, as 15 potências relativas $\left(R P_{\delta, i}, R P_{\theta, i}, R P_{\alpha, i}, R P_{\beta, i}\right.$ e $R P_{\gamma, i}$, com $i=$ $\{\max$, min, med $\})$ foram calculadas dos 20 segmentos de todos os 19 canais. Uma média de cada potência relativa foi obtida ao longo de cada canal, resultando em 15 potências relativas médias por canal. Esses valores médios foram utilizados como parâmetros para discriminar, por canal, dentro dos seguintes pares de grupos de estudo: $\mathrm{C}$ vs $\mathrm{MCI}, \mathrm{C}$ vs $\mathrm{ADM}$, $\mathrm{C}$ vs ADA, MCI vs ADM, MCI vs ADA e ADM vs ADA.

\section{A. Seleção de Parâmetros}

Como os valores das potências relativas dependem da wavelet utilizada na decomposição, uma primeira busca foi realizada para encontrar a wavelet que resulta em parâmetros (potências relativas médias por canal) com maior capacidade discriminante para cada par de grupos de estudo. As famílias de wavelets avaliadas foram Biortognais, Biortogonais Reversas, Daubechies, Coiflets, Symlets e Fejer-Korovkin.

Após calcular os parâmetros utilizando todas as wavelets avaliadas, eles foram separados por cada combinação de par de grupos, canal e wavelet. E, para cada combinação, eles foram aplicados ao classificador binário X-ROC [17]. Esse classificador utiliza a distância quadrática entre médias de distribuições para encontrar um bom limiar para discriminar duas classes e a ROC para analisar o resultado da classificação [17].

Para cada combinação de par de grupos e canal, selecionouse os parâmetros que resultaram em acurácias de classificação iguais ou superiores a $85 \%$. Ressalta-se que um parâmetro pode ter sido selecionada mais de uma vez, onde em cada uma foi obtido com uma wavelet diferente.

Como mencionado anteriormente, a discriminação dentro dos pares de grupos de estudo será realizada por canal. Assim, para cada combinação de par de grupos de estudo e canal, os parâmetros pré-selecionados foram aplicados a um algoritmo genético de forma a selecionar o melhor conjunto contendo até 75 parâmetros.

\section{B. Classificação Utilizando Parâmetros Selecionados}

Para cada combinação de par de grupos de estudo e canal, o melhor conjunto de características foi utilizado como entrada de classificadores binários. Quatro classificadores foram avaliados, nomeadamente, árvores de decisão (DT, do inglês Decision Trees), análise discriminante linear (LDA, do inglês Linear Discriminant Analysis), e máquinas de vetor suporte (SVM, do inglês Support Vector Machines) linear (L) e quadrática (Q). Em todos os casos, para verificar a capacidade de generalização dos classificadores, uma validação cruzada leave-one-out foi utilizada.

Os resultados de classificação para cada combinação de par de grupos de estudo, canal e classificador estão indicados nos mapas topográficos exibidos na Figura 1. A acurácia máxima dos canais e os eletrodos onde foi obtida assim como a acurácia média em todo o escalpe estão destacadas.

Na discriminação dentro do par C vs MCI, acurácias de $100 \%$ foram alcançadas em 2 eletrodos nos classificadores DT, SVM quadrático e LDA. Os eletrodos onde isso ocorreu foram F8, Fp2, P4, O2 e Cz, com destaque para Fp2 por ter obtido essa acurácia ideal em 2 classificadores e a máxima acurácia de $93,80 \%$ no SVM linear. De forma geral, as regiões com maiores diferenças significativas foram o lóbulo frontal direito, ambos os lóbulos temporais, o lóbulo occipital e o lóbulo parietal posterior. As classificações médias ficaram entre 82,4 e 85,5\%, com a maior sendo obtida com LDA.

No caso do par C vs ADM, acurácias de $100 \%$ foram alcançadas em pelo menos 1 eletrodo também nos classificadores DT, SVM quadrático e LDA. Os eletrodos onde isso ocorreu foram P3, P4, T3, Fp1 e Fp2, com destaque para P3 e T3 por terem obtido essa acurácia ideal em 2 classificadores. De forma geral, as regiões com maiores diferenças significativas foram os lóbulos parietal, esquerdo temporal e frontal anterior esquerdo. As diferenças entre esses dois grupos estão mais espalhadas pelo escalpe que no caso anterior. O classificador LDA destacou-se por obter $100 \%$ de acurácia em 4 eletrodos. As classificações médias ficaram entre 86,50 e 88,20\%, com a maior sendo obtida com DT e SVM linear.

Em relação ao par C vs ADA, acurácias de $100 \%$ foram alcançadas em pelo menos 2 eletrodos com todos os classificadores. Os eletrodos onde isso ocorreu foram $\mathrm{O} 1, \mathrm{~F} 3, \mathrm{~F} 4$, F7, F8, Fp1, Fp2, C4, T5 e T6, com destaque para Fp1, Fp2 e F3 por terem obtidos essa acurácia ideal em 3 classificadores. Assim como no caso $\mathrm{C}$ vs $\mathrm{ADM}$, há diferenças significativas entre os grupos por todo o escalpe. Mas as maiores diferenças estão no lóbulo frontal esquerdo e na área temporal-occipital direita. Novamente, o classificador LDA destacou-se por obter $100 \%$ em 7 eletrodos. As classificações médias ficaram entre 88,50 e $91,40 \%$, com a maior obtida justamente com LDA.

No estudo do par MCI vs ADM, acurácias de 100\% foram alcançadas em pelo menos 1 eletrodo com todos os classificadores. Os eletrodos onde isso ocorreu foram F4, F8, $\mathrm{Fz}, \mathrm{C} 4, \mathrm{Cz}$ e $\mathrm{O} 2$, com destaque para F8 por ter obtido essa acurácia ideal em 3 classificadores. Em geral, as diferenças significativas entre os grupos estão localizadas nos lóbulos temporal anterior direito, temporal posterior esquerdo, frontal direito e occipital direito. O classificador LDA destacou-se 


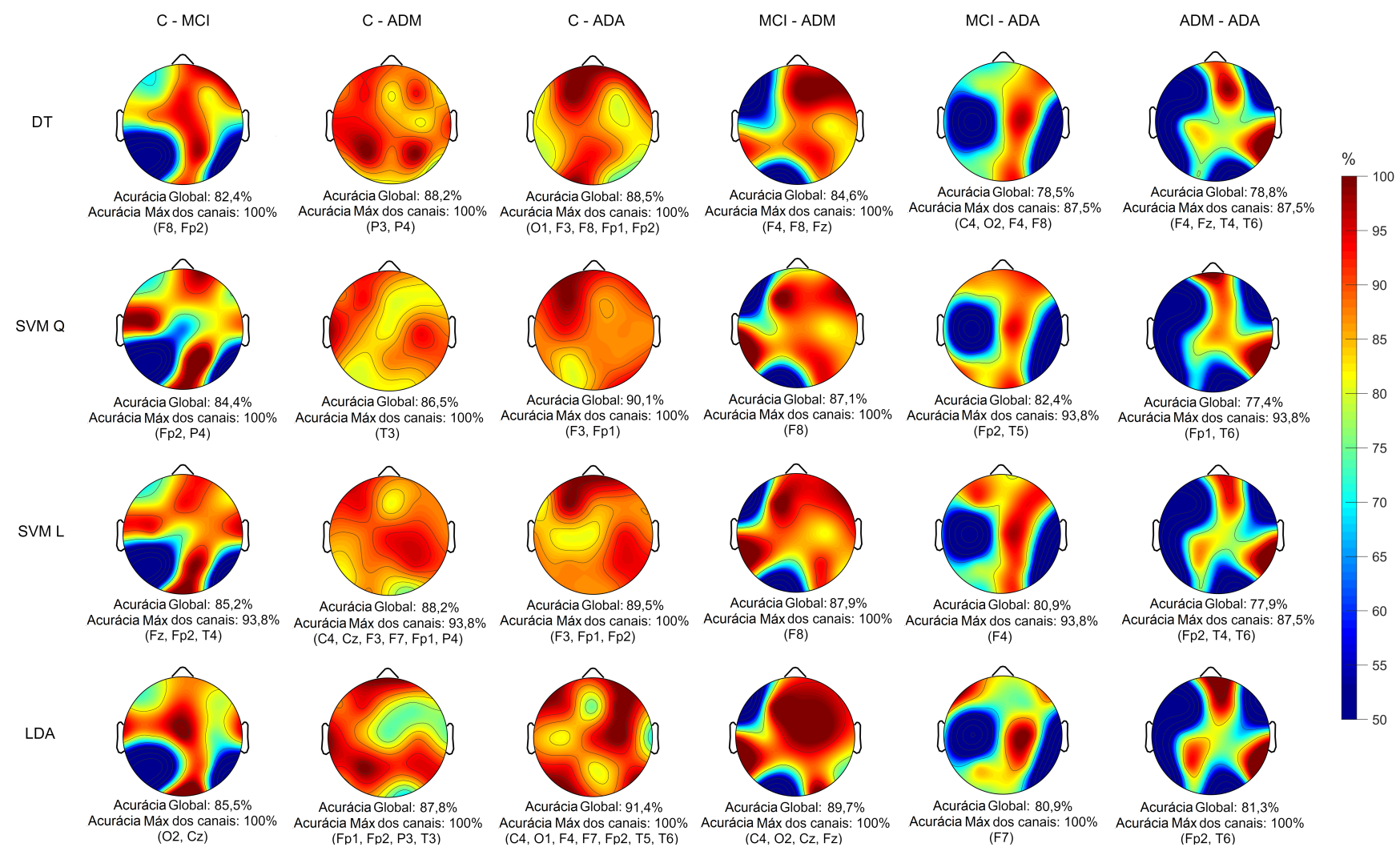

Fig. 1. Mapas topográficos das classificações obtidas entre pares de grupos de estudo ao nível dos eletrodos.

mais uma vez por atingir $100 \%$ de acurácia em 4 eletrodos. As classificações médias ficaram entre 84,60 e $89,70 \%$, onde a maior foi obtida justamente com LDA.

No caso MCI vs ADA, $100 \%$ de acurácia foi alcançado apenas no eletrodo F7 com o classificador LDA. Nos outros classificadores, as maiores acurácias foram $87,50 \%$ nos eletrodos C4 O2, F4 e F8 com DT, 93,80\% nos Fp2 e T5 com SVM quadrático, e 93,80\% no F4 com SVM linear. Em geral, as maiores diferenças entre os grupos foram obtidas nos lóbulos parietal central direito e frontal anterior. As classificações médias ficaram entre 78,85 e $82,40 \%$, onde o pior caso foi obtido com DT e o melhor com SVM quadrático.

Por fim, em relação ao par ADM vs ADA, 100\% de acurácia foi alcançado apenas nos eletrodo Fp2 e T6 com o classificador LDA. Nos outros classificadores, as maiores acurácias foram $87,50 \%$ nos eletrodos F4, Fz, T4 e T6 com DT, 93,80\% nos Fp1 e T6 com SVM quadrático, e 87,50\% no Fp2, T4 e T6 com SVM linear. Em geral, as maiores diferenças entre os grupos foram obtidas nos lóbulos frontal anterior e temporal posterior direito. As classificações médias ficaram entre 77,40 e $81,30 \%$, onde o pior caso foi obtido com SVM quadrático e o melhor com LDA.

De forma geral, os resultados demonstraram que a combinação de potências relativas de sinais de EEG obtidas através da Wavelet Packet é adequada para detectar diferenças significativas no escalpe entre diferentes estágios da DA. Os eletrodos onde se obteve as maiores diferenças entre os pares de grupos foram F4, F8, Fp1, Fp2 e T6. Esses eletrodos estão localizados nos lóbulos frontal e temporal, áreas do escalpe associadas à
TABELA II

COMPARAÇÃO DAS MAIORES ACURÁCIAS MÉdIAS COM O ESTADO DA ARTE.

\begin{tabular}{c|cc}
\hline \multirow{2}{*}{ Trabalho } & \multicolumn{2}{|c}{ Pares de Grupos de Estudo } \\
\cline { 2 - 3 } & C-MCI & C-ADM \\
\hline \hline$[19]$ & - & $86 \%$ \\
{$[20]$} & - & $84 \%$ \\
{$[21]$} & - & $75 \%$ \\
{$[22]$} & - & $90 \%$ \\
{$[23]$} & $93 \%$ & - \\
{$[24]$} & - & $94 \%$ \\
{$[16]$} & $77 \%$ & $95 \%$ \\
{$[25]$} & $88 \%$ & - \\
Presente & $85,5 \%$ & $88,2 \%$ \\
\hline
\end{tabular}

memória e funções cognitivas [18]. E uma análise do escalpe na sequência $\mathrm{C}$ vs $\mathrm{MCI}$, MCI vs ADM e ADM vs ADA fornece uma indicação das regiões no escalpe com maiores diferenças à medida que a doença progride continuamente.

A comparação dos maiores resultados médios obtidos neste trabalho com os métodos do estado da arte para detectar DA através de sinais EEG é ilustrada na Tabela II. A comparação é baseada nos resultados médios porque os trabalhos do estado da arte mencionados utilizam informações provenientes de todos os eletrodos. Observa-se que, entre os pares de grupos de estudo analisados neste trabalho, apenas discriminações dentro dos pares C vs MCI e C vs ADM foram encontradas na literatura. Isso deve-se ao grande foco dado na detecção precoce da DA. Outros trabalhos encontrados na literatura realizam detecções entres outros pares de grupos, por exemplo 


\section{$\mathrm{C}$ vs $\mathrm{ADM}+\mathrm{ADA}$ ou $\mathrm{C}$ vs $\mathrm{MCI}+\mathrm{ADM}$.}

No que se refere à $\mathrm{C}$ vs $\mathrm{MCI}$ e $\mathrm{C}$ vs $\mathrm{ADM}$, observa-se que o método proposto apresentou acurácias médias de classificação melhores que alguns trabalhos encontrados na literatura mas não superou o estado da arte. No entanto, deve-se atentar que as acurácias médias apresentadas neste trabalho levam em consideração todos os eletrodos. Como baixas acurácias foram obtidas em alguns eletrodos devido aos classificadores não encontrarem diferenças significativas nas potências relativas entre os grupos naquela localização do escalpe, as acurácias médias são atenuadas. Se apenas alguns canais forem considerados, em particular aqueles onde acurácias de $100 \%$ foram obtidas, as acurácias médias de classificação obtidas neste trabalho muito provavelmente aumentariam.

\section{Conclusões}

Este trabalho investigou a detecção da doença de Alzheimer em diferentes estágios através de sinais EEG. A detecção foi realizada entre pares de grupos de estudo e por eletrodo. Potências relativas às frequências convencionais foram obtidas dos valores máximo, mínimo e médio de estimativas do espectro de potências da Wavelet Packet. Para cada par de grupos de estudo e eletrodo, uma seleção dos parâmetros foi realizada utilizando classificador binário e algoritmo genético.

Os parâmetros selecionados foram utilizados como entrada para 4 classificadores binários, nomeadamente, DT, LDA, e SVM linear e quadrática, com validação cruzada leave-oneout. Acurácias de classificação de $100 \%$ foram obtidas, em pelo menos 1 eletrodo, para os 6 pares de grupos analisados, indicando as regiões no escalpe com maiores diferenças à medida que a doença progride. E acurácias médias, incluindo todos os eletrodos, entre 81,3 e $91,4 \%$ foram alcançadas. O classificador LDA apresentou o melhor desempenho geral.

\section{AGRADECIMENTOS}

Os autores gostariam de agradecer à Unidade de Neurologia do Hospital de São João, Porto, Portugal, pelo fornecimento da base de dados. Esse trabalho foi apoiado pelos Fundos Nacionais da Fundação para a Ciência e a Tecnologia (FCT), Portugal, através do projeto UIDB/50016/2020.

\section{REFERENCIAS}

[1] C. Ballard, S. Gauthier, A. Corbett, C. Brayne, D. Aarsland, and E. Jones, "Alzheimer's disease," The Lancet, vol. 377, pp. 1019-1031, 2011.

[2] T. Vos, C. Allen, M. Arora, R. M. Barber, and A. Brown et al. "Global, regional, and national incidence, prevalence, and years lived with disability for 310 diseases and injuries, 1990-2015: a systematic analysis for the Global Burden of Disease Study 2015," The Lancet, vol. 388, no. 10053, pp. 1545-1602, 2016.

[3] A. M. Hurtado-Puerto, C. Russo, and F. Fregni, "Alzheimer's Disease," in Neuromethods, 2018, ch. 9, pp. 297-338.

[4] M.-T. Heemels, "Neurodegenerative diseases," Nature, vol. 539, no. 7628, pp. 179-179, November 2016.

[5] R. Cassani, M. Estarellas, R. San-Martin, F. J. Fraga, and T. H. Falk, "Systematic Review on Resting-State EEG for Alzheimer's Disease Diagnosis and Progression Assessment," Disease Markers, vol. 2018, pp. 1-26, October 2018.

[6] N. Houmani, F. Vialatte, E. Gallego-Jutglà, G. Dreyfus, V.-H. NguyenMichel, J. Mariani, and K. Kinugawa, "Diagnosis of Alzheimer's disease with Electroencephalography in a differential framework," PLOS ONE, vol. 13, no. 3, pp. 1-19, 2018.
[7] T. Tong, Q. Gao, R. Guerrero, C. Ledig, L. Chen, D. Rueckert, and A. D. N. Initiative, "A novel grading biomarker for the prediction of conversion from mild cognitive impairment to alzheimer's disease," IEEE Transactions on Biomedical Engineering, vol. 64, no. 1, pp. 155165, January 2017.

[8] J. Jeong, "EEG dynamics in patients with Alzheimer's disease," Clinical Neurophysiology, vol. 115, no. 7, pp. 1490-1505, 2004.

[9] J. Weller and A. Budson, "Current understanding of Alzheimer's disease diagnosis and treatment," F1000Research, vol. 7, 2018.

[10] M. Galea and M. Woodward, "Mini-Mental State Examination (MMSE): Commentary," Australian Journal of Physiotherapy, vol. 51, no. 3, p. 198, 2005.

[11] M. Tanveer, B. Richhariya, R. U. Khan, A. H. Rashid, P. Khanna, M. Prasad, and C. T. Lin, "Machine learning techniques for the diagnosis of alzheimer's disease," ACM Transactions on Multimedia Computing, Communications, and Applications, vol. 16, no. 1s, pp. 1-35, apr 2020

[12] S. Sanei, EEG signal processing. Chichester, England Hoboken, NJ: John Wiley \& Sons, 2007.

[13] M. Vetterli and J. Kovačević, Wavelets and Subband Coding. Englewood Cliffs, New Jersey: Prentice Hall, 1995.

[14] M. V. Wickerhauser, "Lectures on wavelet packet algorithms," in Lecture notes, INRIA, 1991, pp. 31-99.

[15] O. Rioul and M. Vetterli, "Wavelets and signal processing," IEEE Signal Processing Magazine, vol. 8, no. 4, pp. 14-38, October 1991.

[16] P. M. Rodrigues, J. P. Teixeira, C. Garrett, D. Alves, and D. Freitas, "Electroencephalogram Signal Analysis in Alzheimer's Disease Early Detection," International Journal Of Reliable and Quality E-Healthcare, vol. 7, no. 1, pp. 40-58, 2018.

[17] C. T. Nakas and C. T. Yiannoutsos, "Ordered multiple-class ROC analysis with continuous measurements," Statistics in Medicine, vol. 23, no. 22, pp. 3437-3449, November 2004.

[18] K. H. Jawabri and S. Sharma, Physiology, Cerebral Cortex Functions, 2019.

[19] C. Besthorn, H. Forstl, C. Geiger-Kabisch, H. Sattel, T. Gasser, and U. Schreiter-Gasser, "Eeg coherence in alzheimer's disease," Electroencephalogr Clin Neurophysiol, vol. 90, pp. 242-245, 1994.

[20] C. Huang, L. Wahlund, T. Dierks, P. Julin, B. Winblad, and V. Jelic, "Discrimination of alzheimer's disease and mild cognitive impairment by equivalent eeg sources: a cross-sectional and longitudinal study," Clinical Neurophysiology, vol. 111, pp. 1961-1967, 2000.

[21] V. Knott, E. Mohr, C. Mahoney, and V. Ilivitsky, "Quantitative electroencephalography in alzheimer's disease: comparison with a control group, population norms and mental status," J Psychiatr Neurosci, vol. 26, pp. 106-116, 2001.

[22] A. Petrosian, D. Prokhorov, W. Lajara-Nanson, and R. Schiffer, "Recurrent neural network-based approach for early recognition of alzheimer's disease in eeg," Clinical Neurophysiology, vol. 112, pp. 1378-1387, 2001.

[23] F. Vialatte, A. Cichocki, G. Dreyfus, T. Musha, S. Shishkin, and R. Gervais, "Early detection of alzheimer's disease by blind source separation, time frequency representation, and bump modeling of eeg signals," Lecture Notes in Computer Science, vol. 3696, pp. 683-692, 2005.

[24] C. Melissant, A. Ypma, E. Frietman, and C. Stam, "A method for detection of alzheimer's disease using ica-enhanced eeg measurements," Artificial Intelligence in Medicine, vol. 33, pp. 209-222, 2005.

[25] S. Khatun, B. I. Morshed, and G. M. Bidelman, "A single-channel EEG-based approach to detect mild cognitive impairment via speechevoked brain responses," IEEE Transactions on Neural Systems and Rehabilitation Engineering, vol. 27, no. 5, pp. 1063-1070, May 2019. 